\title{
Influence of Polymer Crystallinity and Morphology on Positron Annihilation Characteristics
}

\author{
M. DȨBOWSKA \\ Institute of Experimental Physics, University of Wrocław \\ pl. M. Borna 9, 50-204 Wrocław, Poland
}

Some most representative results for semicrystalline polymers by positron annihilation spectroscopy method, showing the influence of crystallinity and morphology of samples on positron annihilation characteristics, are reminded. The latest finding of the author's experiment on modified polyamide 6 is presented. The latter reveals that the interfacial zone between a lamella and an amorphous region may influence the ortho-positronium characteristics. The role of chain defects is stressed.

PACS numbers: 36.10.Dr, 78.70.Bj, 81.30.Hd, 82.35.Lr

\section{Introduction}

The characteristics of the longest-lived component(s) in the positron annihilation lifetime spectra (PALS), ascribed to ortho-positronium (o-Ps) atoms decays in free-volume holes, are used to get information on the concentration of free-volume holes, the mean size of the holes, and the fractional free volume. Carrying out such studies of semicrystalline polymers one must be sure about the role of crystalline domains in positron annihilation mechanism. However, the question of influence of the presence of crystalline regions on ortho-positronium annihilation characteristics, although having been studied and announced since years, has not been solved yet. Some representative examples, of the attempts undertaken in the past, are given in the paper to show that there is no general relationship between the level of crystallinity and the characteristics. The examples of the influence of morphology of samples on o-Ps annihilation characteristics are included, although very few of such studies are known from literature. However, not always any correlation of the characteristics with crystallinity and morphology of samples was observed.

\section{Examples of influence of crystallinity on positron annihilation characteristics}

In the very early papers the information was already given that in positron lifetime spectra measured for polymers the long-lived component appeared of con- 
siderable intensity. The exemplary data for semicrystalline polytetrafluoroethylene (PTFE) and polyethylene (PE) from [1] are presented in Table I.

TABLE I

Characteristics of the long-lived component for PTFE and PE [1].

\begin{tabular}{c|c|c|c}
\hline \hline Sample & $\begin{array}{c}\text { Temperature } \\
{\left[{ }^{\circ} \mathrm{C}\right]}\end{array}$ & $\begin{array}{c}\tau_{2} \\
{[\mathrm{~ns}]}\end{array}$ & $\begin{array}{c}I_{2} \\
{[\%]}\end{array}$ \\
\hline \multirow{3}{*}{$\mathrm{PTFE}$} & 20 & $3.5 \pm 0.4$ & $\approx 30$ \\
& -78 & $2.5 \pm 0.4$ & $\approx 30$ \\
& -196 & $1.6 \pm 0.4$ & $\approx 30$ \\
\hline $\mathrm{PE}$ & 20 & $2.4 \pm 0.3$ & $29 \pm 5$
\end{tabular}

Then, with the improvement of the method of analysis, three-component fit occurred to be better and two long-lived components were usually extracted from the positron lifetime spectra, as in [2]. Exemplary results for semicrystalline polyamide 6 (PA6) and polypropylene (PP) are in Table II.

\section{TABLE II}

Characteristics of the two long-lived components revealed in the three-component analysis of the positron lifetime spectra for PA6 and PP [2].

\begin{tabular}{c|c|c|c|c}
\hline \hline \multirow{2}{*}{ Sample } & \multicolumn{2}{|c|}{ 2nd component } & \multicolumn{2}{c}{ 3rd component } \\
\cline { 2 - 5 } & $\tau_{2}[\mathrm{~ns}]$ & $I_{2}[\%]$ & $\tau_{3}[\mathrm{~ns}]$ & $I_{3}[\%]$ \\
\hline PA6 & $0.68 \pm 0.10$ & $5.5 \pm 2.0$ & $1.71 \pm 0.08$ & $15.6 \pm 1.0$ \\
PP & $0.84 \pm 0.10$ & $4.4 \pm 1.5$ & $2.50 \pm 0.10$ & $21.4 \pm 1.0$
\end{tabular}

Authors [2] assumed the sum of the intensities $\left(I_{2}+I_{3}\right)$ as the total intensity of the $o$-Ps, decaying via pick-off in crystalline and amorphous domains of the sample, respectively. The possibility of $o$-Ps quenching, when bounded with a molecule in the crystalline domains, was taken into account, too. For PTFE the sum became smaller when sample crystallinity increased, as it is shown in Table III.

\section{TABLE III}

Influence of sample crystallinity on the intensity of $o$-Ps components in the lifetime spectra for PTFE [2].

\begin{tabular}{c|c}
\hline \hline Heat treatment & $I_{2}+I_{3}[\%]$ \\
\hline quenching & 33 \\
not used & 29 \\
annealing & 22
\end{tabular}


The authors' [2] general conclusion was that positron mean lifetime may be different in substances of the same chemical content but of different structure. Because an increase in $I_{2}$ was observed with crystallinity, the proposal was given to take the ratio $\frac{I_{2}}{I_{2}+I_{3}}$ as a measure of the crystallinity of the studied samples. Similar trend (a rise of the ratio with crystallinity of PTFE) was found in [3] but it occurred not to be the general rule (cf. results for poly(butadiene) in the same paper [3]).

The improvement of both experimental techniques and the way of analysis of the positron lifetime spectra resulted in still greater number of lifetime components being revealed in the PALS spectra. In [4] three, of the four lifetime components found in the spectra of semicrystalline PE, were ascribed to annihilation events in three different regions of the studied samples, i.e. lamellae, less ordered regions between lamellae and amorphous regions between spherulites. By chance the crystallinity of the samples from differential scanning calorimetry (DSC) and X-ray diffraction technique was numerically close to the sum of relative intensities of positron annihilation in lamellae and between lamellae. It confirmed the authors in their belief about a connection between positron annihilation characteristics and the crystallinity of samples.

In [5] the linear correlation between the percentage of crystallinity and the longest-lived component intensity $I_{3}$, assigned to $o$-Ps localized in free-volume sites, was observed for polyetheretherkethone (PEEK). According to the results, the $o$-Ps intensity would be zero in a single crystal of PEEK, while the observed $o$-Ps lifetime was constant, regardless of the degree of crystallinity. In opinion of the authors [5] the small thickness of the crystalline lamellae (about $2.0 \mathrm{~nm}$ ) allowed all of the $o$-Ps to diffuse to free-volume sites.

In contrary to the results of [5], the intensities of the two long-lived components $\left(I_{3}, I_{4}\right)$ were both independent of crystallinity of samples of the semicrystalline syndiotactic polystyrene [6]. Quick diffusion (in a fraction of a picosecond) of $o$-Ps formed in the crystalline region was this time assumed to explain the independence of the intensities of crystallinity.

The decrease in $I_{3}$ (the longest-lived component intensity) with increasing crystallinity was reported in [7] for semicrystalline polyethylene terephthalate $(\mathrm{PET})$. However, the extrapolation gave $I_{3} \approx 6 \%$ at $100 \%$ crystallinity. Therefore, the conclusion was that the semicrystalline phase in PET contributed to the $o$-PS formation. According to the authors' hypothesis [7] the inhomogeneous electron distribution existing in the crystal structure of PET is the reason that "even small lattice distortions, such as long range thermal vibrations with frequencies $<10^{8} \mathrm{~Hz}$ can provide trapping sites for $o$-Ps formation in the crystalline phase in the regions of reduced atomic/electron density, and the respective $I_{3}$ dependence on the crystallinity should be linear." The above hypothesis was checked by the same authors in studies of the temperature dependence of $I_{3}[7]$. As far as the result of [5] is concerned, the opinion was given [7] that such intra-crystalline trapping sites 
for $o$-Ps formation are simply not present in the PEEK crystal because of a more uniform atomic/electronic density distribution, in comparison to PET molecules.

Other results of PALS studies of highly crystalline polymers (PET, PE 90-95\% vol.) [8] demonstrate that formation of $o$-Ps in the crystalline phase is more abundant at high temperatures and, in opinion of the authors, not necessarily correlated to the availability of free-volume sites. They assume that positronium formation and annihilation take place in thermally generated $o$-Ps localization sites within the crystalline lattice, which exhibit relatively long lifetimes ( $>2 \mathrm{~ns})$. The probability of positronium formation should depend, in that case, on the vibrational properties of the material since the amount of these sites changes reversibly with the temperature [8].

\section{Examples of morphology influence on positron annihilation characteristics}

In [9] PE samples obtained by isothermal crystallization, with well defined lamellar structure, and the content of defects as well, were studied. The aim of the investigation was to find if any correlation exists between the mechanism of positron annihilation and the microstructure of the studied PE samples. The general conclusion was that the annihilation characteristics may be related to the average distance between crystallites, thickness of them, and the concentration of chain defects. The research was then continued and in [10] a model of positronium diffusion in semicrystalline PE was given. Positronium was assumed to be formed in crystalline and amorphous phases and its tunneling through the interface between both phases was proposed. Although the ideas of the positronium diffusion and escape from the crystalline to the amorphous phase are acceptable, the model in [10] is incorrect. As it was shown in [11], the expression for the positronium transition probability $P$ from the crystalline to amorphous phase, given in [10] should be substituted with the formula (1)

$$
P=\frac{2 L_{1}}{l_{\mathrm{c}}} \tanh \left(\frac{l_{\mathrm{c}}}{2 L_{1}}\right),
$$

where $l_{\mathrm{c}}$ and $L_{1}$ denote the thickness of the lamella and the positronium diffusion length, respectively. The mistake resulted in four times greater value of the positronium diffusion length, $L_{1}$, fitted in [10] to the obtained results. The correct value of $L_{1}$ is $2.5 \mathrm{~nm}$. The resultant positronium flux may be really pointed at a broad amorphous domain between thin lamellae, especially when the positronium diffusion length in the lamellae $L_{1}$ is greater than the one in the amorphous region $L_{\mathrm{a}}$. Very low values of $L_{\mathrm{a}}$ (a few $\AA$ at room temperature (RT)) were in fact reported in [12] for some amorphous polymers. In [13] a large difference was found between the positronium diffusion coefficient in semicrystalline ice (about $0.2 \mathrm{~cm}^{2} / \mathrm{s}$ ) and in amorphous ice (roughly $10^{-3} \mathrm{~cm}^{2} / \mathrm{s}$ ). Trapping of positronium in defects in the amorphous ice was assumed as the reason of the large difference. In contrast to the results given in $[9,10]$ no correlation of $o$-Ps characteristics with 
such morphological parameters as the long period and the lamella thickness was found in [7]. More data concerning PALS studies of semicrystalline polymers can be found in [14]. Comprehensive studies of semicrystalline PE [15] and PTFE [16] are worthy to be mentioned, too.

\section{The interfacial zone in polymers}

An existence of a third, interfacial phase in semicrystalline polymers, that can make up to $20 \%$ of the material, is evidenced by results of dielectric relaxation, NMR, small-angle neutron scattering, specific heat measurements, electron microscopy, and analysis of the Raman internal modes [17]. The main reason of the existence of the interfacial phase is the difference in conformation of chains in the crystalline and amorphous domains of semicrystalline polymers. It is assumed that the interfacial zone is characterized by a partial ordering of chains. In [18] a model of such zone, given by Struik, is mentioned.

According to the model: "the crystalline regions disturb the amorphous phase and reduce its segmental mobility. This reduction is at its maximum in the immediate vicinity of the crystallites; at large distances from the crystallites the properties of the amorphous phase will become equal to those of the bulk amorphous material" [18]. More detailed characterization of the interfacial zone can be found in [17].

\section{Results for modified polyamide 6}

5.1. Polyamide 6 - general information

Polyamide 6 is a polymer with two crystalline forms $(\alpha$ and $\gamma)$. The $\alpha$-form has a monoclinic structure, where neighboring chains form hydrogen bonds, which in turn generate a planar hydrogen-bonded sheet (ac-plane). These sheets stack together via van der Waals interactions. This structure gives two interchain diffraction signals at spacings of $0.44 \mathrm{~nm}$ (indexed as (200)) and $0.37 \mathrm{~nm}$ (indexed as $(020) /(220))$, respectively. The single sheets generate a (002) diffraction signal from the planes of $\mathrm{C}=\mathrm{O}$ groups at a spacing of $0.862 \mathrm{~nm}$. The $\gamma$-form also has a monoclinic structure, but because the amide units tilt and rotate out of the $a c$-plane, hydrogen bonds occur between parallel chains, which explain a reduction of the two interchain distances in the region $0.40-0.42 \mathrm{~nm}$. The hydrogen bonding is poorer in the $\gamma$-form compared to the $\alpha$-form.

\subsection{Properties of the studied samples}

As a result of the way of preparation described in detail in [19] three samples, denoted further as: PA6 raw, $\mathrm{PA} 6 \mathrm{H}_{2} \mathrm{O}$ and PA6 LN, were obtained. The symbol PA6 $\mathrm{H}_{2} \mathrm{O}$ denotes a sample after modification of the PA6 raw with water vapor at high temperature and pressure, while PA6 LN refers to a sample obtained as a result of quenching the PA6 raw sample with liquid nitrogen. The results of studies of the modified samples, obtained by wide-angle X-ray scattering (WAXS) and small-angle X-ray scattering (SAXS), are summarized in Table IV. 
Characteristics of the samples from WAXS and SAXS studies.

\begin{tabular}{c|c|c|c|c|c|c|c|c}
\hline \hline Sample & $\begin{array}{c}\text { Crystallinity } \\
{[\mathrm{wt.} \%]}\end{array}$ & $\begin{array}{c}\alpha \text {-form } \\
{[\mathrm{wt. \%}]}\end{array}$ & $\begin{array}{c}\gamma \text {-form } \\
{[\mathrm{wt. \%}]}\end{array}$ & $\begin{array}{c}D_{\alpha(200)} \\
{[\mathrm{nm}]}\end{array}$ & $\begin{array}{c}D_{\gamma(200)} \\
{[\mathrm{nm}]}\end{array}$ & $\begin{array}{c}L \\
{[\mathrm{~nm}]}\end{array}$ & $\begin{array}{c}l_{\mathrm{c}} \\
{[\mathrm{nm}]}\end{array}$ & $\begin{array}{c}E \\
{[\mathrm{~nm}]}\end{array}$ \\
\hline PA6 raw & 46.1 & 38.7 & 7.4 & 3.3 & 3.7 & 6.3 & 2.9 & 1.5 \\
PA6 LN & 46.2 & 34.8 & 11.4 & 2.7 & 5.4 & 6.2 & 2.5 & 1.5 \\
PA6 $\mathrm{H}_{2} \mathrm{O}$ & 44.1 & 36.3 & 7.8 & 10.7 & 12.1 & 8.4 & 3.2 & 0.8
\end{tabular}

$D_{\alpha(200)}$ and $D_{\gamma(200)}$ in Table IV denote the mean crystallite size in the direction perpendicular to the (200) planes of the both polymorphic forms, $L$ is the long period (the average distance between the crystallites, along the chain axis), $l_{\mathrm{c}}$ is the average thickness of the lamella, $E$ is the average thickness of the transition layer between the lamella and the amorphous region. In the transition zone the number density of the electronic charge was assumed to change linearly from $\rho_{\mathrm{c}}$, characteristic of the lamella, to $\rho_{\mathrm{a}}-$ characteristic of the amorphous domain. Crystallinity of the studied samples is the same (one must take into account the error $\approx 1.5 \mathrm{wt} . \%$ ) but the morphology changed a little as a result of the modification. Quenching resulted in a higher content of the $\gamma$-form (less dense and less stable in a thermodynamic sense) in the PA6 LN sample. SAXS curves [19] indicate that the best and most uniform lamellar structure exists in the sample PA6 $\mathrm{H}_{2} \mathrm{O}$, in which the thickness of the amorphous layer is the highest, while the transition layer between semicrystalline and amorphous domain is the thinnest one. In the same sample the crystallites are the biggest ones. When comparing the WAXS curves for PA6 $\mathrm{H}_{2} \mathrm{O}$ and PA6 LN in the range of $2 \Theta$ from $16^{\circ}$ to $28^{\circ}$, as it is presented in Fig. 1 in [20], one obtains the following information:

- Positions of the peaks $\alpha(200)$ and $\alpha(020) /(220)$, corresponding to the $\alpha$-crystalline form, are in case of the sample PA6 $\mathrm{H}_{2} \mathrm{O}$ very close to the theoretical ones. The peak coming from the plane (200) corresponds to the interchain distance in the plane where the hydrogen bonds between the groups $\mathrm{CO}$ and $\mathrm{NH}$ exist.

- In case of the sample PA6 LN distinct shifts of the $\alpha$-peaks take place: (1) to the higher values of $2 \Theta$ in the case of the left peak (the shift denotes the decrease in the interchain distance), (2) to the smaller scattering angles in case of the right peak (enlargement of the interchain distance). The shifts suggest both the deformation of the unit cell of the $\alpha$-crystalline form in the quenched sample and the presence of defects.

\subsection{Results of positron annihilation lifetime spectra measurements}

A description of the measurements as well as the way of the analysis of the PALS spectra were given in [19]. Here, the information on the $o$-Ps characteristics is only delivered. In Figs. 1 and 2 respectively, the intensity $I_{3}$ and the average 
value, $\left\langle\tau_{3}\right\rangle$, of the log-normal distribution of the lifetimes corresponding to the longest-lived component, are presented for the samples of the most different morphology, i.e. PA6 LN and PA6 $\mathrm{H}_{2} \mathrm{O}$, above the glass transition temperature of the sample PA6 raw.

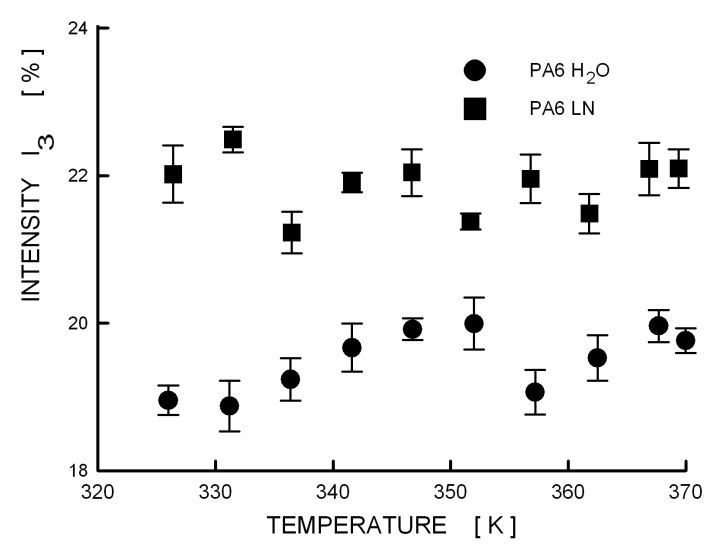

Fig. 1. The temperature dependence of the longest-lived component intensity $I_{3}$.

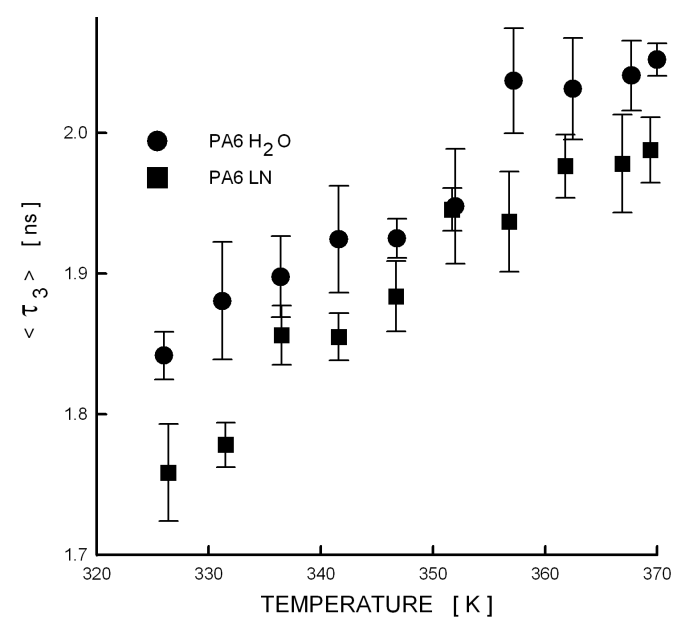

Fig. 2. The temperature dependence of the average value, $\left\langle\tau_{3}\right\rangle$, of the log-normal distribution of the lifetimes corresponding to the longest-lived component.

It is seen that $I_{3}$ is distinctly higher for the sample PA6 LN than for the PA6 $\mathrm{H}_{2} \mathrm{O}$ sample. Taking into account that the density of the $\gamma$-phase is about $6 \%$ smaller than the density of the $\alpha$-phase, the higher content of the $\gamma$-form may result in the increase in the intensity of the longest-lived component in the quenched sample. It is connected with the looser packing of PA6 chains in part of the crystalline regions of the sample PA6 LN containing more $\gamma$-crystalline form than the 
sample PA6 $\mathrm{H}_{2} \mathrm{O}$. Specific volume of amorphous zones in polyamide 6 depends on the ratio of the $\alpha$-form to the $\gamma$-form content [21]. Higher content of $\gamma$-form results in higher specific volume of the amorphous zones. In Fig. 3 the average value of the determined $o$-Ps intensity $\left(I_{3 \mathrm{av}}\right)$, in the whole region of the temperature above the glass transition temperature, is given for all the studied samples vs. the reciprocal of the long period $L$ (lamellar periodicity from SAXS). $L^{-1}$ values are proportional to the number density of the interfaces (lamella/transition zone) where different defects (i.e. jogs, loops, chain ends, dislocations) may exist.

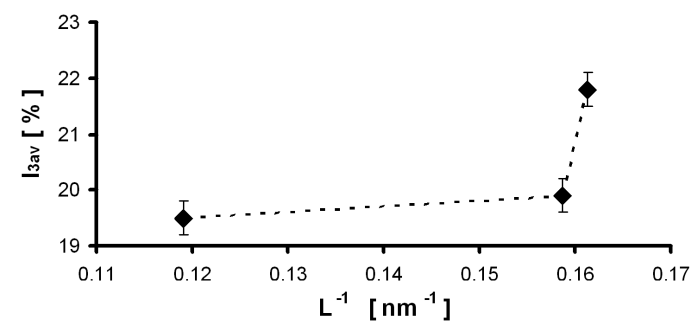

Fig. 3. The influence of the number density of the interfacials on the $o$-Ps intensity.

The rise in $I_{3 a v}$ values with the density of the interfaces points out the important role of the interfaces and defects at them in the process of ortho-positronium annihilation (cf. Fig. 3 in [9]). The highest value of $I_{3 \mathrm{av}}$ for the sample PA6 LN corresponds well with both the deformation of the unit cell of the $\alpha$-form and the presence of defects found by the shift of the peaks of $\alpha$-form in the WAXS curve for this sample.

In Fig. 2 the values of $\left\langle\tau_{3}\right\rangle$ for the PA6 $\mathrm{H}_{2} \mathrm{O}$ sample are arranged a little higher in comparison to those for PA6 LN. One may suspect that it is the result of the lesser topological constraints imposed by the presence of crystallites on the chains in the amorphous zones of the $\mathrm{PA} 6 \mathrm{H}_{2} \mathrm{O}$ in which the zones are the broadest ones. The lesser topological constraints mean the looser packing of the chains in the central parts of the amorphous zones which may contribute to the increase in the $\left\langle\tau_{3}\right\rangle$ values for the sample PA6 $\mathrm{H}_{2} \mathrm{O}$.

\section{Discussion and summary}

Special treatment of the raw sample of the PA6 with water vapor and liquid nitrogen resulted in changes of morphology of the samples. The changes, although not as great as expected, were detected by WAXS and SAXS methods.

As it was shown in Sect. 2 of this paper, the influence of morphology on positron annihilation mechanism in semicrystalline polymers, is not clear. It seems that the formation and annihilation of positronium in both the crystalline and amorphous phases depends on the material under investigation. To be sure that the ortho-positronium characteristics reflect only the free volume properties of the amorphous phase of the semicrystalline polymers, one should carry out thorough 
investigation of the studied samples. Especially, apart from the crystallinity level and morphological data (the long period, thickness of the lamella and the transition layer), the distribution, type, and size of defects should be known.

Not having information on defects in the studied samples, but taking into account the data and results presented in Sect. 5, one may suspect that:

- Positronium is formed in the crystalline and the amorphous domains as well as in the transition zone of the samples of polyamide 6 .

- Positronium annihilates in the whole volume of the samples. The diffusion of $o$-Ps may take place from the crystalline to the amorphous domains (especially in case of thin lamellae and thin transition zones), but it is disturbed by trapping $o$-Ps atoms in chain defects. The values of the positronium transition probability $(P)$ from the lamella to the transition zone, according to the formula (1) for the studied samples are: 0.90 (PA6 raw), 0.92 (PA6 LN), and $0.88\left(\mathrm{PA} 6 \mathrm{H}_{2} \mathrm{O}\right)$, when using the correct value of $L_{1}$ (i.e. $2.5 \mathrm{~nm}$ ) for $\mathrm{PE}$ from [10] and the $l_{\mathrm{c}}$ values from Table IV. Corresponding values of $P$ when the escape from the interfacial zone to the amorphous domain is concerned are: 0.97 (PA6 raw and PA6 LN), $0.99\left(\mathrm{PA} 6 \mathrm{H}_{2} \mathrm{O}\right)$ using the same value of $L_{1}$ and the values of $E$ from Table IV. Although the value of $L_{1}$ for PA6 is not known, it is reasonably to accept it to be close to that of $\mathrm{PE}$, and use it in order to estimate the values of $P$. Taking into account the estimated values of $P$ one can see that the escape to the amorphous zone is the most probable in case of the sample PA6 LN, thus contributing to the increase in the value of $I_{3}$ for this sample.

- Defects in the crystalline and in the transition zones are probably characterized by the lifetimes smaller but close to the lifetime characteristic of the free-volume holes in the amorphous domains in the studied samples of polyamide 6 . One may take into account such defects as chain loops, chain end clusters (the so-called "amorphous defects") [9] well known and established in PE. The defects contribute to the unoccupied volume of the whole samples.

- The contribution of the $o$-Ps decays in defects is not possible to be extracted as an individual lifetime component. The longest-lived component intensity $I_{3}$ is probably a mixture coming from the $o$-Ps annihilation events in the defects and in the free-volume holes. As a proof one may take a little shorter value of $\left\langle\tau_{3}\right\rangle$ for the PA6 LN sample with defected $\alpha$-crystalline form in comparison to the value for the $\mathrm{PA} 6 \mathrm{H}_{2} \mathrm{O}$ sample in which the best crystalline structure was detected.

- The higher values of $\left\langle\tau_{3}\right\rangle$ in case of the sample PA6 $\mathrm{H}_{2} \mathrm{O}$ may reflect also the weaker influence of the crystalline phase on the amorphous domains (especially on the central zones of them, according to Struik's model), which are the broadest ones among the studied samples. 
The results indicate that positron annihilation studies of PA6 are particularly complex and need more extensive and systematic studies, for more numerous set of well defined and more differentiated samples, to elucidate the question of the contribution of the crystallinity and morphology of samples.

\section{Acknowledgments}

This work was supported by University of Wrocław, grant no. 2016/IFD/06.

\section{References}

[1] R.E. Bell, R.L. Graham, Phys. Rev. 90, 644 (1955).

[2] S. Tao, J.H. Green, Proc. Phys. Soc. 85, 463 (1965).

[3] J.R. Stevens, M.J. Edwards, J. Polym. Sci. C 30, 297 (1968).

[4] J.R. Stevens, P.C. Lichtenberg, Phys. Rev. Lett. 29, 166 (1972).

[5] H. Nakanishi, Y.C. Jean, J. Polym. Sci., Part B: Polym. Phys. 27, 1419 (1989).

[6] B.G. Olson, T. Prodpran, A.M. Jamieson, S. Nazarenko, Polymer 43, 6775 (2002).

[7] L. Xie, D.W. Gidley, H.A. Hristov, A.F. Yee, Polymer 35, 14 (1994).

[8] H.A. Hristov, A.F. Yee, D.W. Gidley, http://flux.aps.org/meetings/YR9596/BAPSMAR96/abs/S260009.html.

[9] J. Balta Calleja, J. Serna, J. Vicente, M.A. Segovia, J. Appl. Phys. 58, 253 (1985).

[10] J. Serna, Phys. Status Solidi A 122, 489 (1990).

[11] W. Świątkowski, Nukleonika 48, 141 (2003).

[12] K. Hirata, Y. Kobayashi, Y. Ujihira, J. Chem. Soc. Faraday Trans. 92, 985 (1996).

[13] M. Eldrup, A. Vehanen, P.J. Schultz, K.G. Lynn, Phys. Rev. 32, 7048 (1985).

[14] P.E. Mallon, in: Principles and Applications of Positron and Positronium Chemistry, Eds. Y.C. Jean, P.E. Mallon, D.M. Schrader, World Scientific, Singapore 2003, p. 253.

[15] G. Dlubek, J. Stejny, Th. Lupke, D. Bamford, K. Petters, Ch. Hübner, M.A. Alam, M.J. Hill, J. Polym. Sci., Part B: Polym. Phys. 40, 65 (2002).

[16] G. Dlubek, A. Sen Gupta, J. Pionteck, R. Häßler, R. Krause-Rehberg, H. Kaspar, K.H. Lochhaas, Polymer 46, 6075 (2005).

[17] L. Mandelkern, in: Physical Properties of Polymers, Ed. J.E. Mark, American Chemical Society, Washington 1993, p. 175.

[18] D.W. van Krevelen, Properties of Polymers, 3rd ed., Elsevier Science B.V., Amsterdam 1990, p. 32.

[19] M. Dȩbowska, J. Pigłowski, Cz. Ślusarczyk, P. Schmidt, J. Rudzińska-Girulska, T. Suzuki, R. Yu, W. Biniaś, Fibres Text. East. Eur. 13 5(53), 64 (2005).

[20] M. Dȩbowska, J. Pigłowski, C. Ślusarczyk, J. Rudzińska-Girulska, T. Suzuki, R. Yu, Radiat. Phys. Chem., in press.

[21] G. Gurato, A. Fischera, F.Z. Grandi, R. Zanetti, P. Canal, Die Makromol. Chem. 175, 953 (1974). 